\title{
Introduction International Institute for Archival Science, Trieste-Maribor in 2015
}

\author{
Peter Pavel KLASINC, Ph.D. \\ Archival councillor, Head: Departement of Archives and Records Management at Alma Mater Europaea, ECM, \\ Director: International Institute for Archival Science of Trieste/Maribor \\ e-mail: peter.klasinc@guest.arnes.it
}

There is no doubt that the decision of the IIAS T/M one year ago, here in Trieste, was correct, as we decided for themes that have attracted both regular members of the Institute as well as some researchers of archival theory and practice from all over the world.

Over and over again we, in the Institute (this year we are celebrating the $30^{\text {th }}$ anniversary of the Institute's establishment and its functioning *1985), we want to follow the opportunities offered by our conference, which are the current exchange of experiences and transfer of the knowledge, and especially the development of archival theory and practice as well as of the archival science in all geographical areas, where archives, which are indisputably important cultural, scientific and educational institutions, operate.

We know that the archives of the world more or less successfully perform their basic mission, which is the storage and preservation of archival records as the written cultural heritage and the memory of nations.

At the same time, let me remind you again on our ARCHIVAL CODE and the DECLARATION ON ARCHIVES, where we find the basis for this year's themes of our conference, because the contemporary problems of management, evaluation and long-term preservation of archival records are topical themes and are in the position of the real interdependence with the issues concerning archival buildings, their design and decisions on new construction or renovation and interior decorating questions. Besides all this, a particularly important role represent the optimum solution regarding the premises for the storage of archival and current records, and the equipment as well as the operation of archival service with qualified professionals at creators of archival records, where the professional archival theory and practice indisputably start and thus the archival science develop.

The International Institute for Archival Science, Trieste - Maribor in the time from November 2014 to October 2015 was acting within the framework of the mission and opportunities determined by the statute. The Executive Committee (Grazia Tatò, Antonio Monteduro, Peter Pavel Klasinc, Zdenka Semlič Rajh) regularly held, and with the support of Claudia Salminen and Carmela Bianca in recent period successfully operated. Mauro Tosti Croce as a Chairman of the General Meeting of the members of the Institute and a representative of the Ministry of Cultural Property in Rome, due to the departure to a new duty at the beginning of the year left us. To its place stepped Gulia Barrera. The Institute receives traditional support by Higher education institute Alma Mater Europaea - European Centre Maribor, led by President Prof. Dr. Ludvik Toplak. This educational institute a few years ago began to implement a post-graduated study of archival science and record management and this caused that Alma Mater also took over some publishing and other obligations of the IIAS T/M. For number of years we are also receiving the support from the Agency for Research of the Republic of Slovenia in Ljubljana (Prof. Dr. József Györkös) and the Centre of European Initiatives (Antonio Monteduro). Institute for many years creates a multilingual dictionary of archival terminology and has implemented many international archival contacts especially with regular or future members of the Institute from Slovenia, Italy, Austria, Germany, France*, Croatia, Czech Republic, Slovakia, Montenegro, Israel*, Canada, Russia, Ukraine, Bosnia and Herzegovina, Belarus, Spain, Poland, Romania, Greece, Estonia, Malaysia, Hungary, Macedonia and the Sultanate of Oman. Countries *Kazakhstan, *Vietnam and England are in the process of the member appointment (a total of 27 countries). I am 
especially pleased that we have been joined by a large number of visiting lecturers, excellent archival experts from all parts of the world. The Institute's library has received around 20 books, which are with previous received books on the list for registration in the system COBISS or adequate Italian version.

Good contacts are continuing with ICA from Paris and its Secretary General David Leitch, with the National Archives of the Sultanate of Oman, where we have performed educational course in February. Only with the help of our sponsors we were able to perform some of our activities.

The Institute has prepared the International Archival Day, $25^{\text {th }}$ issue of the journal for contemporary archival theory and practice, ATLANTI 2015 Volume 1 and 2 and The $9^{\text {th }}$ Autumn Archival School. A whole week (from $18^{\text {th }}$ to $25^{\text {th }}$ October 2014) the city of Trieste is the scene of international archival professional events: our Conference, Atlanti Magazine, archival school, meetings with sponsors, etc. The regular conference of members of the Institute will also be held (present will be around 20 countries), throughout all that week time Autumn archive school will take place, in which 25 young archivists from all parts of the world will be trained.

At the $25^{\text {th }}$ Conference International Archival Day and the issue of $25^{\text {th }}$ issue of ATLANTI I am thanking all former and current regular members of the Institute, many lecturers and unselfish colleagues in institutions, where the Institute operates.

Special thanks to employees at the State Archives in Trieste, Director Dr. Claudia Salmini, Dr. Grazia Tatò and Carmelo Bianco and all the paper contributors, the translators of texts in Atlanti and to those who help us at the conference to overcome language barriers and to all those who have supported the activities of our Institute.

This year, I am especially thankig the affection of Alma Mater Europaea - the European Centre Maribor, companies Scope of Switzerland, In Vision DIO Austria, KARTONAŽA Vlašić from Croatia and Mikrografija from Slovenia.

I see no reason, at the end, not to call once again: “ATLANTI - Avanti (Forward)"!

\section{Introduzione I'Istituto Internazionale per le Scienze Archivistiche di Trieste e Maribor nel 2015}

Non c'è dubbio che la decisione dell'Istituto Internazionale per le scienze Archivistiche di Trieste e Maribor (IIAS T/M) presa un anno fa, qui a Trieste, sia stata corretta, decidendo di scegliere temi che hanno attirato sia i membri regolari dell'Istituto che ricercatori di teoria e pratica archivistica da tutto il mondo.

Ancora una volta noi, nell'Istituto (quest'anno si celebra il trentesimo anniversario della fondazione dell'Istituto e del suo funzionamento * 1985), vogliamo seguire le opportunità offerte dalla nostra conferenza, che sono lo scambio di esperienze ed il trasferimento di conoscenze e soprattutto lo sviluppo della teoria e pratica archivistica in tutte le aree geografiche dove esistono gli archivi, che sono indiscutibilmente importanti istituzioni culturali, scientifiche ed educative,.

Sappiamo che gli archivi del mondo svolgono più o meno correttamente la loro missione di base, che consiste nel deposito e nella conservazione del materiale archivistico come eredità culturale scritta e memoria delle Nazioni.

Allo stesso tempo, permettetemi di ricordarvi ancora una volta il nostro Codice archivistico e la nostra Dichiarazione sugli archivi, dove troviamo la base per i temi di quest'anno della nostra conferenza, perché i problemi contemporanei della gestione, valutazione e conservazione a lungo termine del materiale archivistico sono temi d'attualità e sono realmente interdipendenti con le questioni che riguardano gli edifici d'archivio, le loro scelte su nuove costruzioni o ristrutturazioni e l'allestimento 
degli interni ed il design. Oltre a tutto questo, un ruolo particolarmente importante riguarda la soluzione ottimale per quanto dei locali per il deposito dell'archivio storico e corrente e le attrezzature, nonché il funzionamento del servizio di archiviazione effettuato da professionisti qualificati e dai soggetti produttori del materiale archivistico, momenti nei quali si dà avvio all'archiviazione professionale così come allo sviluppo della scienza archivistica.

Nel periodo da novembre 2014 a ottobre 2015 l'Istituto Internazionale per le Scienze Archivistiche di Trieste e Maribor ha agito nell'ambito della missione e delle opportunità stabilite dallo statuto. Il Comitato esecutivo (Grazia Tatò, Antonio Monteduro, Peter Pavel Klasinc, Zdenka Semlič Rajh) si è regolarmente riunito, con il supporto di Claudia Salmini e di Carmelo Bianco. A causa dell'assegnazione ad altro incarico nell'ambito dell'Amministrazione archivistica italiana di Mauro Tosti Croce, presidente dell'Assemblea generale dei membri dell'Istituto e rappresentante del Ministero dei beni culturali al suo posto è subentrata Gulia Barrera. L'Istituto riceve tradizionalmente supporto dall'Istituto di istruzione superiore Alma Mater Europaea di Maribor, centro europeo, guidato dal Presidente Dr. Ludvik Toplak. Questo istituto educativo da pochi anni ha cominciato ad implementare un corso post-laurea di scienza archivistica e gestione documentale, e ciò ha comportato che l'Alma Mater ha in parte supportato l'Istituto Internazionale per le Scienze Archivistiche di Trieste e Maribor. Per diversi anni abbiamo anche ricevuto il supporto dell'Agenzia per la ricerca della Repubblica di Slovenia a Lubiana (Dr. József Györkös) e dell'Iniziativa Centro Europea (Antonio Monteduro). Da molti anni l'Istituto ha redatto un dizionario multilingue di terminologia archivistica ed ha implementato molti contatti internazionali con istituzioni archivistiche, soprattutto con regolari o futuri membri dell'Istituto da Slovenia, Italia, Austria, Germania, Francia*, Croazia, Repubblica Ceca, Slovacchia, Montenegro, Israele *, Canada, Russia, Ucraina, Bosnia-Erzegovina, Bielorussia, Spagna, Polonia, Romania, Grecia, Estonia, Malesia, Ungheria, Macedonia e Sultanato dell'Oman. Paesi come * Kazakistan, * Vietnam e Inghilterra sono in procinto di essere nominati membri (per un totale di 27 Paesi). Sono particolarmente contento che si è unito a noi un gran numero di docenti, eccellenti esperti d'archivistica da tutte le parti del mondo. La biblioteca dell'Istituto ha ricevuto circa 20 libri, che con le recenti acquisizioni sono in lista per la registrazione nel sistema COBISS o analoga versione italiana.

Buoni contatti stanno continuando con l'ICA di Parigi ed il suo segretario generale David Leitch, con gli archivi nazionali del Sultanato dell'Oman, dove abbiamo effettuato corsi formativi in febbraio. Solo con l'aiuto dei nostri sponsor, siamo stati in grado di eseguire alcune delle nostre attività.

L'Istituto ha preparato la Giornata archivistica internazionale, il numero 25 della rivista per la teoria e pratica archivistica contemporanea, ATLANTI 2015 Volume 1 e 2 e la IX scuola archivistica d'autunno. Per un'intera settimana (dal 18 al 25 ottobre 2014) la città di Trieste è stata scena di eventi professionali archivistici internazionali: la nostra conferenza, Atlanti Magazine, la Scuola di archivistica, incontri con sponsor, ecc. Si terrà inoltre l'annuale conferenza dei membri dell'Istituto (rappresentanti circa 20 paesi), e contempraneamente avrà luogo la Scuola, durante la quale saranno formati giovani archivisti provenienti da tutte le parti del mondo.

Per la XXV Giornata archivistica internazionale e la XXV edizione di Atlanti voglio ringraziare i membri dell'Istituto passati e presenti, i molti relatori ed i colleghi delle istituzioni che operano nell'Istituto.

Un grazie particolare ai collaboratori dell'Archivio di Stato di Trieste, al passato direttore dott. Claudia Salmini al nuovo direttore Liliana Bagalà, alla dott. Grazia Tatò, ed a Carmelo Bianco ed a tutti coloro che hanno contribuito alla pubblicazione, ai traduttori dei testi in Atlanti che ci aiutano durante la conferenza a superare le barriere linguistiche, e che hanno sostenuto le attività del nostro Istituto.

Quest'anno mi sento in particolare di ringraziare gli sponsor Alma Mater Europaea - the European Centre Maribor, ditte Scope della Svizzera, In Vision DIO dell'Austria, KARTONAŽA Vlašić dalla Croatia e Mikrografija dalla Slovenia.

E non vedo infine ragione alcuna di non gridare ancora: "ATLANTI - AVANTI!”. 


\section{Uvod Mednarodni inštitut arhivskih znanosti, Trst-Maribor v letu 2015}

Nesporno je, da je bila odločitev članov MIAZ T/M pred enim letom tukaj v Trstu pravilna, saj smo se odločili za teme, ki so pritegnile tako redne člane inštituta, kakor tudi nekatere raziskovalce arhivske teorije in prakse iz vseh delov sveta.

Vedno znova želimo v inštitutu (letos slavimo 30. letnico obstoja oz. delovanja *1985) slediti možnostim, ki jih nudi naša konferenca; to je tekoče izmenjavati izkušnje in prenašati znanja, predvsem pa razvijati arhivsko teorijo in prakso, kakor tudi arhivsko znanost v vseh geografskih prostorih, kjer delujejo arhivi, ki so nesporno pomembne kulturne, znanstvene in izobraževalne ustanove.

Vemo, da arhivi po svetu več ali manj uspešno opravljajo svojo osnovno poslanstvo, hranjenje in varovanje arhivskega gradiva kot pisne kulturne dediščine in spomin narodov.

Ob tem mi dovolite, da vas znova spomnim na naš ARHIVSKI KODEKS in na DEKLARACIJO O ARHIVIH, kjer najdemo osnove za letošnje teme naše konference, saj so sodobni problemi vodenja, vrednotenja in dolgo dobnega varovanja arhivov zelo aktualni in so v dejanski soodvisnosti od problemov v zvezi z arhivskimi zgradbami, njihovim oblikovanjem in odločitvami o novogradnjah ali adaptacijah, ter vprašanji notranjega opremljanja. Ob vsem tem imajo posebej pomembno vlogo optimalne rešitve prostorov za hrambo arhivskega in dokumentarnega gradiva in njihova oprema ter delovanje arhivskih služb z usposobljenimi arhivskimi strokovnjaki pri ustvarjalcih, kjer se nesporno prične profesionalna arhivska teorija in praksa in se tako razvija tudi arhivska znanost.

Mednarodni inštitut arhivskih znanosti, Trst-Maribor je v času od novembra 2014 do oktobra 2015 deloval v okviru poslanstva in možnosti, ki jih določa statut. Redno je zasedal izvršni komite (Grazia Tatò, Antonio Monteduro, Peter Pavel Klasinc, Zdenka Semlič Rajh), ki je s podporo Claudije Salmini in Carmela Bianca tudi v preteklem obdobju uspešno deloval. Mauro Tosti Croce nas je kot predsednik skupščine članov inštituta in predstavnik Ministrstva za kulturne dobrine iz Rima, zaradi odhoda na novo dolžnost v začetku leta zapustil. Na njegovo mesto je stopila Gulia Barrera. Inštitut je deležen tradicionalne podpore, Visokošolske ustanove Alma Mater Europaea - Evropski center Maribor, ki jo vodi predsednik prof. dr. Ludvik Toplak. Na tej ustanovi se je pred leti pričel izvajati magistrski študij arhivistike in dokumentologije in to je bil vzrok, da je Alma Mater prevzela tudi del izdajateljskih in nekaterih drugih obveznosti MIAZ T/M. Podpore smo že vrsto let deležni od Agencije za raziskovalno dejavnost R. Slovenije iz Ljubljane (prof. dr. József Györkös ) in od Centra evropskih iniciativ (Antonio Monteduro). Institut že vrsto let izdeluje večjezični slovar arhivske terminologije ter izvaja mnoge mednarodne arhivske stike predvsem z rednimi ali bodočim člani inštituta iz Slovenije, Italije, Avstrije, Nemčije, Francije *, Hrvaške, Češke, Slovaške, Črne Gore, Izraela*, Kanade, Rusije, Ukrajine, Bosne in Hercegovine, Belorusije, Španije, Poljske, Romunije, Grčije, Estonije, Malezije, Madžarske, Makedonije in Sultanata Oman. Države * Kazahstan, * Vietnam in *Anglija so v postopku imenovanja uradnega člana (skupno 27 držav).

Posebej sem vesel, da se nam je pridružilo veliko število gostujočih predavateljev, izvrstnih arhivskih strokovnjakov iz vseh delov sveta. Knjižnica inštitut je prejela okoli 20 knjig, ki z že prej prejetimi knjigami še vedno čakajo na vpis v sistem COBISS oz. primerljivo italijansko verzijo.

Dobri stiki se nadaljujejo z MAS iz Pariza in generalnim sekretarjem Davidom Leitchem, z Nacionalnim arhivom iz Sultanata Oman, kjer smo v februarju izvedli izobraževalno konferenco. Le $s$ pomočjo sponzorjev pa smo lahko izvedli nekatere naše aktivnosti.

Inštitut je pripravil Mednarodni arhivski dan, 25. številko Revije za sodobno arhivsko teorijo in prakso, ATLANTI 2015 zvezek 1 in 2 ter pripravili 9. Jesensko arhivsko šolo. Mesto Trst je ves teden (od 18. do 25.10.2014) prizorišče mednarodnih arhivskih strokovnih dogodkov t.j. naša konferenca, revija Atlanti, arhivska šola, srečanja s sponzorji itd. Opravljena bo redna konferenca članov inštituta (prisotnih bo okoli 20 držav), ves čas pa bo potekala Jesenska arhivska šola, na kateri se bo usposabljalo 25 mladih arhivistk in arhivistov iz vseh delov sveta. 
Peter Pavel KLASINC: Introduction International Institute for Archival Science, Trieste-Maribor in 2015, 25-29

Ob 25. konferenci Mednarodni arhivski dan in ob izdaji XXV (25) številke ATLANTOV se najlepše zahvaljujem vsem nekdanjim in sedanjim rednim članom inštituta, mnogim predavateljem in požrtvovalnim sodelavcem $\mathrm{v}$ ustanovah, kjer inštitut deluje.

Hvala zaposlenim v Državnem arhivu v Trstu, direktorici dr. Claudii Salmini in dr. Grazii Tatò ter Carmelu Biancu ter vsem avtorjem prispevkov, prevajalcem tekstov v Atlantih in tistim, ki nam na konferenci pomagajo premagovati jezikovne pregrade ter vsem tistim, ki so podprli aktivnosti našega inštituta.

Letos se posebej zahvaljujem za naklonjenost Almi Mater Europaei - Evropskemu centru Maribor, podjetjem Scope iz Švice, In Vision D.I.O. iz Avstrije, Kartonaži Vlašič iz Hrvaške in Mikrografiji iz Slovenije.

Ne vidim razloga, da ne bi na koncu ponovno zaklical "ATLANTI - avanti (naprej)” 